\title{
Cosmological dynamics of $R^{n}$ gravity
}

\author{
S Carloni†, P K. S. Dunsby $\ddagger$ S Capozziello§, A Troisi $\|$ \\ $\dagger \ddagger$ Department of Mathematics and Applied Mathematics, University of Cape Town, \\ South Africa. \\ ¥ South African Astronomical Observatory, Observatory Cape Town, South Africa. \\ $\S$ Dipartimento di Scienze Fisiche e Sez. INFN di Napoli, Universita' di Napoli \\ "Federico II", Complesso Universitario di Monte S. Angelo, Via Cinthia I-80126 \\ Napoli (Italy) \\ || Dipartimento di Fisica "E. R. Caianiello", Università di Salerno, I-84081 Baronissi, \\ Salerno e Istituto Nazionale di Fisica Nucleare, sez. di Napoli, Gruppo Collegato di \\ Salerno, Via S. Allende-84081 Baronissi (SA), Italy
}

\begin{abstract}
.
A detailed analysis of dynamics of cosmological models based on $R^{n}$ gravity is presented. We show that the cosmological equations can be written as a first order autonomous system and analyzed using the standard techniques of dynamical system theory. In absence of perfect fluid matter, we find exact solutions whose behavior and stability are analyzed in terms of the values of the parameter $n$. When matter is introduced, the nature of the (non-minimal) coupling between matter and higher order gravity induces restrictions on the allowed values of $n$. Selecting such intervals of values and following the same procedure used in the vacuum case, we present exact solutions and analyze their stability for a generic value of the parameter $n$. From this analysis emerges the result that for a large set of initial conditions an accelerated expansion is an attractor for the evolution of the $R^{n}$ cosmology. When matter is present a transient almost-Friedman phase can also be present before the transition to an accelerated expansion.
\end{abstract}

PACS numbers: 98.80.Jk, 04.50.+h, 05.45.-a 


\section{Introduction}

At the present time, there are many good reasons to consider General Relativity (GR) as our best theory for the gravitational interaction. It accounts for the shortcomings of Newtonian gravity and fits beautifully all the tests that we have been able to devise at laboratory and Solar System levels. Nevertheless, in the last few decades, there is more and more evidence that GR may be incomplete. In particular, the difficulties to match GR with quantum theory, the flattening of galactic rotation curves and, more recently, the cosmic acceleration can be considered hints that some aspects of gravitational interaction are still unknown.

It is for these reasons that much recent effort has been spent on the study of generalizations of the Einstein theory and in particular the so-called Extended Theory of Gravitation (ETG).

One of the most interesting classes of ETG, called non-linear gravity theories or higher-order theories of gravity, is based on gravitational actions which are non-linear in the Ricci curvature $(R)$ and/or contain terms involving combinations of derivatives of $R$ 1, 2, 3. The higher order theories of gravity arise in a wide range of different frameworks. For example, it can be shown that for quantum field theory in curved spacetime, the renormalization of the stress energy tensor implies the introduction of higher order corrections to the GR gravitational action [4]. In addition, the low energy limit of $D=10$ superstring theory is a theory of gravity with additive higher order corrections to the Hilbert-Einstein (HE) term [5, 6]. Finally, the general form of the vacuum action for Grand Unification Theories (GUT) is a higher order theory and the GUT models obtained by relaxing the constraint of linear gravity have, in general, better physical properties than the standard GR [7].

Unfortunately most of the efforts to achieve an insight into the physics of these models has been frustrated by the complexity of the field equations. In fact, the presence of non-linearities and higher order terms makes it hard to achieve both exact and numerical solutions which can be compared with observations. Such difficulties, added to other problems connected with the presence of non-gaugeable ghosts in the particle spectrum of these theories [8], have led researchers to pay more attention towards other alternative gravity theories such as braneworld models 9 .

The recent realization that the universe is currently undergoing an accelerated expansion phase and the quest for the nature of the quintessence field has renewed the interest in higher order theories of gravity and their relation to cosmology. This is due to the fact that the higher order corrections to the HE action can be viewed as an effective fluid and this fluid can emulate the action of the homogeneous part of the quintessence field [10]. Hence, in this Curvature Quintessence scenario, what we observe as a new component of the cosmic energy density is an effect of higher order corrections to the HE Lagrangian. This approach has several advantages over the standard quintessence scenario. For example, we do not need to search for a quintessence scalar field and such theories are more easily constrained by observations. 
A key element of the curvature quintessence scenario is the choice of the form of the Lagrangian density $\mathcal{L}$ for the gravitational interaction. A simple choice is a generic power law of the Ricci scalar $R$. This $R^{n}$-gravity model has been shown to be a good candidate for describing the present evolution of the Universe [10, 11, 12, 13, 14]. Comparison with data coming from supernovae data and the age of the universe as measured by WMAP [15] shows that, at least in the vacuum case, these models are both in agreement with the observations and allow accelerating solutions [16. This result is very important, but incomplete. In fact, we do not know what the stability of these solutions are and which role they play in the global behavior of the underlying cosmological model. In addition, when perfect fluid matter is introduced, the equations become even more complex, making it extremely difficult to find exact solutions.

These problems can be (at least partially) addressed using the dynamical system approach. This powerful method was first developed by Collins and then by Ellis and Wainwright (see [17] for a wide class of cosmological models in the GR context). Some work has also been done in the case of scalar fields in cosmology and for scalartensor theories of gravity [18, 19, 20]. Studying cosmologies using the dynamical systems approach has the advantage of offering a relatively simple method to obtain exact solutions (even if these only represent the asymptotic behavior) and to obtain a (qualitative) description of the global dynamics of these models. Such results are very difficult to obtain by other methods.

The purpose of this paper is to apply the dynamical system approach to higher order gravity and in particular to the $R^{n}$-gravity models. The paper is organized as follows: In section 2, we present the basic equations of the model. In section 3, we analyze dynamics of $R^{n}$-gravity in the vacuum case, find exact solutions and determine their stability. Section 4 gives a generalization of these results to the matter case. Finally, section [5] contains a summary of the results and conclusions.

Unless otherwise specified, we will use natural units $\left(\hbar=c=k_{B}=8 \pi G=1\right)$ throughout the paper, Greek indices run from 0 to 3 and Latin indices run from 1 to 3. The symbol $\nabla$ represents the usual covariant derivative and $\partial$ corresponds to partial differentiation. We use the $(+,-,-,-)$ signature and the Riemann tensor is defined by

$$
R_{\mu \lambda \nu}^{\rho}=W_{\mu \nu, \lambda}^{\rho}-W_{\mu \lambda, \nu}^{\rho}+W_{\mu \nu}^{\alpha} W_{\lambda \alpha}^{\rho}+W_{\mu \lambda}^{\beta} W_{\nu \beta}^{\rho}
$$

where the $W_{\mu \nu}^{\rho}$ is the Christoffel symbol (i.e. symmetric in the lower indices), defined by

$$
W_{\mu \nu}^{\rho}=\frac{1}{2} g^{\rho \alpha}\left(g_{\mu \alpha, \nu}+g_{\alpha \nu, \mu}-g_{\mu \nu, \alpha}\right) .
$$

The Ricci tensor is obtained by contracting the first and the third indices

$$
R_{\mu \nu}=g^{\rho \lambda} R_{\rho \mu \lambda \nu} .
$$




\section{Basic Equations}

If we relax the assumption of linearity in the gravitational action, the general coordinate invariance allows infinitely many additive terms to the HE action:

$$
S=\int d^{4} x \sqrt{-g}\left\{\Lambda+c_{0} R+c_{1} R^{2}+c_{2} R_{\mu \nu} R^{\mu \nu} \ldots+\mathcal{L}_{M}\right\},
$$

where $\mathcal{L}_{M}$ is the matter Lagrangian and we have included corrections up to the fourth order (the fourth order term $R_{\mu \nu \alpha \beta} R^{\mu \nu \alpha \beta}$ has been neglected as a consequence of the Gauss-Bonnet theorem.

The action (41) is not canonical because the Lagrangian function contains derivatives of the canonical variables of order higher than one. This means that, not only do we expect higher order field equations, but also the validity of the Euler-Lagrange equations is compromised.

This problem is particularly difficult in the general case, but can be solved for specific metrics. In fact, once the components of the metric tensor are specified, we can define a new set of generalized coordinates in such a way to reduce formally the degree of the Lagrangian. In these new variables, the higher order field equations correspond to a system of second order differential equations together with a constraint equation associated with the definition of the variables themselves [22]. As will be clear in the following, this procedure is crucial in order to both write the field equations for $R^{n}$ gravity and to apply the dynamical system approach.

In homogeneous and isotropic spacetimes, the Lagrangian in (4) can be further simplified. Specifically, the variation of the term $R_{\mu \nu} R^{\mu \nu}$ can always be rewritten in terms of the variation of $R^{2}[23$. Thus, the "effective" fourth order Lagrangian in cosmology contains only powers of $R$ and we can suppose, without loss of generality, that the general form for a non-linear Lagrangian will be a generic function of the scalar curvature:

$$
L=\sqrt{-g}\left(f(R)+\mathcal{L}_{M}\right) .
$$

By varying $\ddagger$ equation (5), we obtain the fourth order field equations

$$
f^{\prime}(R) R_{\mu \nu}-\frac{1}{2} f(R) g_{\mu \nu}=f^{\prime}(R)^{; \alpha \beta}\left(g_{\alpha \mu} g_{\beta \nu}-g_{\alpha \beta} g_{\mu \nu}\right)+\tilde{T}_{\mu \nu}^{M}
$$

where $\tilde{T}_{\mu \nu}^{M}=\frac{2}{\sqrt{-g}} \frac{\delta\left(\sqrt{-g} L_{M}\right)}{\delta g_{\mu \nu}}$ and the prime denotes a derivative with respect to $R$. It is easy to check that standard Einstein equations are immediately recovered if $f(R)=R$. When $f^{\prime}(R) \neq 0$ the equation (6) can be recast in the more expressive form

$$
G_{\mu \nu}=R_{\mu \nu}-\frac{1}{2} g_{\mu \nu} R=T_{\mu \nu}^{T O T}=T_{\mu \nu}^{R}+T_{\mu \nu}^{M}
$$

where

$$
T_{\mu \nu}^{R}=\frac{1}{f^{\prime}(R)}\left\{\frac{1}{2} g_{\mu \nu}\left[f(R)-R f^{\prime}(R)\right]+f^{\prime}(R)^{; \alpha \beta}\left(g_{\alpha \mu} g_{\beta \nu}-g_{\alpha \beta} g_{\mu \nu}\right)\right\}
$$

$\ddagger$ In the present work we will adopt a "metric approach to derive the field equations. Alternatively, one can use the "Palatini approach" in which the fields " $g$ " and " $W$ " are considered independent 11, 14. 
and

$$
T_{\mu \nu}^{M}=\frac{1}{f^{\prime}(R)} \tilde{T}_{\mu \nu}^{M}
$$

is an effective stress-energy tensor for standard matter. This step is conceptually very important: we have passed from a model in which gravity has a complicated structure to a model in which the gravitational field has the standard GR form with a source made up of two fluids: perfect fluid matter and an effective fluid (curvature fluid) that represents the non-Einsteinian part of the gravitational interaction $\S$.

If we adopt the above picture, there is an important issue related to the conservation properties of the two new fluids. In fact, looking at the Bianchi identities for equation (7), we expect a conservation equation with interaction terms between the curvature fluid and standard matter. However this is not the case. In fact, writing down the Bianchi identities we have

$$
0=T_{\mu \nu}^{T O T ; \nu}=T_{\mu \nu}^{R ; \nu}-\frac{f^{\prime \prime}(R)}{f^{\prime 2}(R)} R^{\nu} \tilde{T}_{\mu \nu}^{M}+\frac{1}{f^{\prime}(R)} \tilde{T}_{\mu \nu}^{M ; \nu} .
$$

Using the field equations and the definition of the Riemann tensor, it is easy to show that the sum of the first two terms of the RHS of the previous expression is zero. Thus, $T_{\mu \nu}^{T O T ; \nu} \propto \tilde{T}_{\mu \nu}^{M}$ and the total conservation equation reduces to the one for matter only. A general, independent proof of this result has been given by Eddington 24] and then by others (like in 25]). They showed that the first variation for the gravitational action is divergence free regardless of the form of the invariant that we choose for the Lagrangian. This means that no matter how complicate the effective stress energy tensor $T_{\mu \nu}^{T O T}$ is, it will always be divergence free if $\tilde{T}_{\mu \nu}^{M ; \nu}=0$. As a consequence, the total conservation equation reduces to the one for standard matter only. This is a very important feature of the curvature fluid and it is the fundamental reason why we can use the Friedmann metric in these models even though the curvature fluid may in general possess heat flux and anisotropic pressure [26].

This paper will focus on a particular higher order model: $R^{n}$-gravity, whose action for the gravitational interaction reads

$$
\mathcal{A}=\int d^{4} x \sqrt{-g}\left[\chi(n) R^{n}+L_{M}\right]
$$

where $\chi(n)$ is a function of $n$ that reduces to 1 for $n=1$. Whatever the value of $n$ we will suppose that the sign of $\chi$ is kept positive in such a way that the attractive character of the gravitational interaction is not compromised.

The field equations for this theory read

$$
\begin{aligned}
& n R^{n-1} G_{\mu \nu}=\chi(n)^{-1} \tilde{T}_{\mu \nu}^{M}+\frac{1}{2} g_{\mu \nu}(1-n) R^{n}+\left[n(n-1) R^{n-2} R^{; \alpha \beta}\right. \\
& \left.+n(n-1)(n-2) R^{n-3} R^{; \alpha} R^{; \beta}\right]\left(g_{\alpha \mu} g_{\beta \nu}-g_{\alpha \beta} g_{\mu \nu}\right)
\end{aligned}
$$

$\S$ Obviously we do not need to restrict ourselves to the $f(R)$ Lagrangians to make this transformation. In general, we could have started with (4) at whatever order and define an effective stress energy tensor in such a way that the field equations have the form (7). 
and for $R \neq 0$ they can be recast as

$$
\begin{aligned}
& G_{\mu \nu}=T_{\mu \nu}^{M}+T_{\mu \nu}^{R}=\frac{\tilde{T}_{\mu \nu}^{M}}{n \chi(n) R^{n-1}}+\frac{1}{2 n} g_{\mu \nu}(1-n) R+\left[(n-1) \frac{R^{; \alpha \beta}}{R}\right. \\
& \left.+(n-1)(n-2) \frac{R^{; \alpha} R^{; \beta}}{R^{2}}\right]\left(g_{\alpha \mu} g_{\beta \nu}-g_{\alpha \beta} g_{\mu \nu}\right) .
\end{aligned}
$$

In this way, the non-Einsteinian part of the gravitational interaction can be modelled as an effective fluid which, in general, posses thermodynamic properties different from standard matter.

In the Friedmann-Lemaitre-Robertson-Walker (FLRW) metric, the above equations can be written as

$$
\begin{aligned}
& 2 n \frac{\ddot{a}}{a}+n(n-1) H \frac{\dot{R}}{R}+n(n-1) \frac{\ddot{R}}{R}+n(n-1)(n-2) \frac{\dot{R}^{2}}{R^{2}}-(1-n) \frac{R}{3} \\
& +\frac{\rho}{3 n \chi(n) R^{n-1}}(1+3 w)=0 \\
& H^{2}+\frac{\kappa}{a^{2}}+H \frac{\dot{R}}{R}(n-1)-\frac{R}{6 n}(1-n)-\frac{\rho}{3 n \chi(n) R^{n-1}}=0 .
\end{aligned}
$$

with

$$
R=-6\left(\frac{\ddot{a}}{a}+\frac{\dot{a}^{2}}{a^{2}}+\frac{\kappa}{a^{2}}\right)
$$

where $H=\dot{a} / a, \kappa$ is the spatial index and we have assumed the standard matter to be a perfect fluid with a barotropic index $w$. Note that in the above equations we have considered $a$ and $R$ as two independent fields. Such a position is standard in canonical quantization of higher order gravitational theories [27] and is very useful in this case because it allows to write the equations (12) as a system of second order differential equations.

The system (14, 16) is completed by the conservation equation for matter, which as we already pointed out, is the same as standard GR, i.e.

$$
\dot{\rho}+3 H \rho(1+w)=0 .
$$

We start first with an analysis of the vacuum case and then present the general case in which matter is present.

\section{The Vacuum Case}

Equations (14) 15) are the starting point of a dynamical analysis of cosmological models based on $R^{n}$ gravity. In vacuum $(\rho=0)$, their form suggests the following choice of expansion normalized variables:

$$
\begin{aligned}
& x=\frac{\dot{R}}{R H}(n-1), \\
& y=\frac{R}{6 n H^{2}}(1-n), \\
& K=\frac{\kappa}{a^{2} H^{2}}=\frac{\kappa}{\dot{a}^{2}} .
\end{aligned}
$$


The variable $x$ is associated with the rate of variation of the Ricci curvature, $y$ represents a measure of the expansion normalized Ricci curvature and $K$ the spatial curvature of the Friedmann model. Following [28, if we consider the Ricci curvature as a scalar field, we can think of $x$ as the kinetic term for this field and $y$ as its potential. However, this analogy does not work completely, because $x$ appears only linearly in the Friedmann equation.

Defining the new time variable $\mathcal{N}=\ln a$, the cosmological equations are equivalent to the autonomous system

$$
\begin{aligned}
x^{\prime} & =2+x-x^{2}+\frac{y}{1-n}(2+n x)+(2+x) K, \\
y^{\prime} & =\frac{x y}{n-1}+2 y\left(\frac{y n}{1-n}+2+K\right), \\
K^{\prime} & =2 K\left(1+\frac{y n}{1-n}+K\right),
\end{aligned}
$$

where the prime represents the derivative with respect to our new "time" coordinate and the dynamical variables are constrained by

$$
1+K+x-y=0 .
$$

It is important to stress that even if the cosmological equations and the dynamical variables are defined for every value of $n$ the dynamical equations (19) are only valid for $n \neq 1$. This is due to the procedure we have used to obtain this system. However, since the case $n=1$ corresponds to standard GR, we will limit our discussion to $n \neq 1$.

\subsection{Finite analysis}

The system (19) can be further simplified because the constraint (20) allows to write the equation for $K$ as a combination of the other two variables $x$ and $y$. In this way, we can reduce (19) to the two dimensional system

$$
\begin{aligned}
& x^{\prime}=(2+x) y-2 x-2 x^{2}-\frac{(2+n x) y}{n-1}, \\
& y^{\prime}=\frac{y}{n-1}[(3-2 n) x-2 y+2(n-1)],
\end{aligned}
$$

and use (20) to determine the value of $K$. Note that if $y=0$ the above system implies $y^{\prime}=0$ and the $x$ axis is an invariant submanifold for the phase space. This means that if the initial condition for the cosmological model is $y \neq 0$ a general orbit can approach $y=0$ only asymptotically. As a consequence, there is no orbit that crosses the $x$ axis and no global attractor can exist.

Setting $x^{\prime}=0$ and $y^{\prime}=0$, we obtain five fixed points

$$
\begin{array}{ll}
\mathcal{A}=\{y \rightarrow 0, x \rightarrow 0\}, & K=-1, \\
\mathcal{B}=\{y \rightarrow 0, x \rightarrow-1\}, & K=0, \\
\mathcal{C}=\left\{y \rightarrow \frac{4 n-5}{2 n-1}, x \rightarrow \frac{2(n-2)}{2 n-1}\right\}, & K=0, \\
\mathcal{D}=\left\{y \rightarrow 2(n-1)^{2}, x \rightarrow-2(n-1)\right\}, & K=2 n^{2}-2 n-1,
\end{array}
$$


Table 1. Coordinates of the fixed points, eigenvalues, and solutions for $R^{n}$-gravity in vacuum.

\begin{tabular}{|c|c|c|}
\hline Point & Coordinates (x,y) & Eigenvalues \\
\hline $\mathcal{A}$ & {$[0,0]$} & {$[-2,2]$} \\
\hline $\mathcal{B}$ & {$[-1,0]$} & {$\left[2, \frac{4 n-5}{n-1}\right]$} \\
\hline $\mathcal{C}$ & {$\left[\frac{2(n-2)}{2 n-1}, \frac{4 n-5}{2 n-1}\right]$} & {$\left[\frac{5-4 n}{n-1}, \frac{4 n+2-4 n^{2}}{2 n^{2}-3 n+1}\right]$} \\
\hline \multirow[t]{6}{*}{$\mathcal{D}$} & {$\left[2(1-n), 2(n-1)^{2}\right]$} & {$[n-2+\sqrt{3 n(3 n-4)}, n-2-\sqrt{3 n(3 n-4)}]$} \\
\hline & Point & Solution \\
\hline & $\mathcal{A}$ & $a=a_{0} t$ \\
\hline & $a=$ & $\iota_{0}\left(t-t_{0}\right)^{1 / 2}($ only for $n=3 / 2)$ \\
\hline & $\mathcal{C}$ & $a=a_{0} t^{\frac{(1-n)(2 n-1)}{n-2}}$ \\
\hline & $\mathcal{D}$ & $\begin{array}{ccc}a=\frac{k t}{2 n^{2}-2 n-1} & \text { if } & k \neq 0 \\
a=a_{0} t & \text { if } & k=0\end{array}$ \\
\hline
\end{tabular}

with $\mathcal{A}$ being a double solution. We can distinguish two classes of fixed point. In the case of the first one $(\mathcal{A}, \mathcal{B})$, the coordinates are independent of the value of $n$ and common to all the $R^{n}$ theories. In the second one $(\mathcal{C}, \mathcal{D})$, the coordinates of the point are $n$-dependent and their position changes with the parameter $n$. In particular, the coordinates of the point $\mathcal{C}$ diverge for $n=1 / 2$.

Merging occurs for the points $\mathcal{C}$ and $\mathcal{B}$ at $n=5 / 4$ and for $\mathcal{C}$ and $\mathcal{D}$ at $n=\frac{1}{2}(1+\sqrt{3})$ and $n=\frac{1}{2}(1-\sqrt{3})$. The points $\mathcal{A}, \mathcal{D}$ would merge at $n=1$, but we will not consider this case because our system is not defined for this value of $n$.

The stability of the fixed points can be obtained by a local stability analysis 30] and this is summarized in Table 2 above.

In $\mathcal{A}$ the linearized matrix has a positive and a negative eigenvalue which is independent of the value of $n$ i.e. it is always a saddle.

The point $\mathcal{B}$ has instead only one fixed (positive) eigenvalue and a second eigenvalue which depends on $n$. Consequently, it is an unstable node for $n<1$ and $n>5 / 4$ and a saddle otherwise.

In the case of $\mathcal{C}$, both the eigenvalues depend on $n$. For $n<\frac{1}{2}(1-\sqrt{3}), 1 / 2<n<1$ and $n>\frac{1}{2}(1+\sqrt{3})$ it corresponds to stable node and for $1<n<5 / 4$ is represents an unstable node. For the other values of $n$, it is a saddle point.

Similarly for $\mathcal{D}$, both the eigenvalues depend on $n$. For $n<\frac{1}{2}(1-\sqrt{3}), n>\frac{1}{2}(1+\sqrt{3})$ it is a saddle point, for $\frac{1}{2}(1-\sqrt{3})<n<0$ and $4 / 3<n<\frac{1}{2}(1-\sqrt{3})$ it is a stable node and for the other values of $n$ the eigenvalues are complex so $\mathcal{D}$ represents an attractive 
Table 2. Stability of the fixed points for $R^{n}$-gravity in vacuum. We refer to the attractive foci as "spirals"

\begin{tabular}{ccccc}
\hline Point & $n<\frac{1}{2}(1-\sqrt{3})$ & $\frac{1}{2}(1-\sqrt{3})<n<0$ & $0<n<1 / 2$ & $1 / 2<n<1$ \\
\hline $\mathcal{A}$ & saddle & saddle & saddle & saddle \\
$\mathcal{B}$ & repulsive & repulsive & repulsive & repulsive \\
$\mathcal{C}$ & attractive & saddle & saddle & attractive \\
$\mathcal{D}$ & saddle & attractive & spiral & spiral \\
\hline
\end{tabular}

\begin{tabular}{ccccc}
\hline Point & $1<n<5 / 4$ & $5 / 4<n<4 / 3$ & $4 / 3<n<\frac{1}{2}(1+\sqrt{3})$ & $n>\frac{1}{2}(1+\sqrt{3})$ \\
\hline $\mathcal{A}$ & saddle & saddle & saddle & saddle \\
$\mathcal{B}$ & saddle & repulsive & repulsive & repulsive \\
$\mathcal{C}$ & repulsive & saddle & saddle & attractive \\
$\mathcal{D}$ & spiral & spiral & attractive & saddle \\
\hline
\end{tabular}

focus $\|$.

A pictorial representation of the phase space in the relevant intervals for $n$ is given in figures (1-8). It is clear that in this plane the $x$ axis represents an invariant submanifold because no orbit crosses it.

The coordinates of the fixed points (22) can be used to find exact solutions for this cosmological model. In fact, by substituting the definitions (18) in the field equations we obtain

$$
\dot{H}=\left(x_{C}+\frac{y_{C}}{n-1}-1\right) H^{2}
$$

which links the coordinates of the fixed point to the form of $H(t)$. If $n \neq 1$ and

$$
(n-1)\left(x_{C}-1\right)+y_{C} \neq 0
$$

this equation can be integrated to give

$$
a=a_{0}\left(t-t_{0}\right)^{\alpha} \quad \text { where } \quad \alpha=\left(1-x_{C}-\frac{y_{C}}{n-1}\right)^{-1} .
$$

For the point $\mathcal{A}$ we have

$$
a=a_{0}\left(t-t_{0}\right),
$$

which represent a Milne evolution and is independent of the value of the parameter $n$. For the point $\mathcal{B}$ we have

$$
a=a_{0}\left(t-t_{0}\right)^{1 / 2},
$$

$\|$ The values of $n$ for which the stability of the fixed points changes are called bifurcations for the system (21). In what follows we will not deal with these specific cases leaving this discussion for a future work. 
but the direct substitution in the equation reveals that this solution is valid only for $n=3 / 2$. For the point $\mathcal{C}$ we have

$$
a=a_{0}\left(t-t_{0}\right)^{\frac{(1-n)(2 n-1)}{n-2}},
$$

which corresponds to a solution obtained elsewhere [10] by direct integration of the field equations. The difference is that the dynamical system approach used here allows us to deduce the stability of this solution. This issue is particularly important when we use the constraint on $n$ from observations [16]. For particular values of this parameter, $\mathcal{C}$ is not only an attractor but also the only finite attractor for the phase space. For the point $\mathcal{D}$ we have again a Milne evolution:

$$
a=a_{0}\left(t-t_{0}\right),
$$

but this time the value of $n$ influences the behavior of the solution. In fact for $n \neq \frac{1 \pm \sqrt{3}}{2}$ the constant $a_{0}$ is given by

$$
a_{0}^{2}=\frac{k}{2 n^{2}-2 n-1},
$$

otherwise the constant $a_{0}$ remains undetermined. It follows that the value of $n$ determines the evolution rate unless $n=\frac{1 \pm \sqrt{3}}{2}$.

It might seem surprising that these solutions posses less than the four integration constants which we would expect when solving a fourth order differential equation. This fact can be explained by noting that the solutions for the fixed points are asymptotic in nature so we can always imagine that the other constants occur in a transient part of the solution that is not relevant in the asymptotic regime.

It is also useful to define the deceleration parameter $q$ in terms of the dynamical variables. We have

$$
q=-\frac{H^{\prime}}{H^{2}}-1=-x-\frac{y}{n-1}
$$

which means, for $q>0$,

$$
\left\{\begin{array} { l } 
{ n > 1 } \\
{ x ( n - 1 ) + y < 0 }
\end{array} \left\{\begin{array}{l}
n<1 \\
x(n-1)+y>0
\end{array}\right.\right.
$$

Thus the phase space is divided into two regions which correspond, once $n$ is fixed, to accelerating and decelerating evolution. It is clear that these conditions are never satisfied for the points $\mathcal{A}, \mathcal{B}, \mathcal{D}$. For the point $\mathcal{C}$ the constraint on $n$ are

$$
\left\{\begin{array}{cc}
n>\frac{1}{2}(1+\sqrt{3}) & \text { expansion } \\
\frac{1}{2}<n<1 & \text { expansion } \\
n<\frac{1}{2}(1-\sqrt{3}) & \text { contraction. }
\end{array}\right.
$$

\subsection{Asymptotic analysis}

Since the phase plane is not compact it is possible that the dynamical system (21) has a nontrivial asymptotic structure. Thus the above discussion would be incomplete without checking the existence of fixed points at infinity (i.e. when the variables 
$x$ or $y$ diverge) and calculating their stability. Physically, such points represents regimes in which one or more of the terms in the Friedmann equation (15) become dominant. For example, if $x \rightarrow \infty$, the "kinetic" part of the curvature energy density dominates over the "potential" part. The asymptotic analysis can easily be performed compactifying the phase space using the so called Poincaré method. In the vacuum case, the compactification can be achieved by transforming to polar coordinates $(r(\mathcal{N}), \theta(\mathcal{N}))$ :

$$
x \rightarrow r \cos (\theta), \quad y \rightarrow r \sin (\theta),
$$

and substituting $r \rightarrow \frac{\mathcal{R}}{1-\mathcal{R}}$ so that the regime $r \rightarrow \infty$ corresponds to $\mathcal{R} \rightarrow 1$. Using the coordinates (34) and taking the limit $\mathcal{R} \rightarrow 1$, the equations (21) can be written as

$$
\begin{aligned}
\mathcal{R}^{\prime} & \rightarrow \frac{(9-8 n) \cos (\theta)-\cos (3 \theta)-7 \sin (\theta)+\sin (3 \theta)}{4(n-1)} \\
\mathcal{R} \theta^{\prime} & \rightarrow \frac{\cos (\theta) \sin (\theta)[\sin (\theta)-\cos (\theta)]}{(n-1)(1-\mathcal{R})} .
\end{aligned}
$$

Starting from (35)|36) we can analyze the asymptotic features of the system (21) in the same way as we did in the finite case. Indeed, since equation (35) does not depend on the coordinate $\mathcal{R}$ we can find the fixed points using only equation (36). Setting $\theta^{\prime}=0$, we obtain six fixed points which are given in Table (3). Their angular coordinate does not depend on the value of the parameter $n$, so they are the same irrespective of the power of $R$ appearing in the initial Lagrangian.

The solutions associated with these fixed points are summarized in Table 3 . The points $\mathcal{A}_{\infty}, \mathcal{D}_{\infty}$ and $\mathcal{C}_{\infty}, \mathcal{F}_{\infty}$ are characterized respectively by the kinetic part or the potential part of the energy density of the curvature fluid dominating the dynamics. Let us derive the solution for $\mathcal{A}_{\infty}$. This point corresponds to $x \rightarrow \pm \infty, y \rightarrow 0$. In the limit $x \rightarrow \infty$ the first equation of the system (21) and (23) reduce to

$$
x^{\prime} \rightarrow-2 x^{2}
$$

and

$$
\frac{H^{\prime}}{H}=x .
$$

Integrating (37) and substituting in (38) we obtain

$$
\left|\mathcal{N}-\mathcal{N}_{\infty}\right|=\left[C_{1} \pm \frac{C_{0}}{2}\left(t-t_{0}\right)\right]^{2},
$$

which represents a scale factor that approaches a constant when $t \rightarrow t_{0}\left(\mathcal{N} \rightarrow \mathcal{N}_{\infty}\right)$. The same procedure can be applied to the points $\mathcal{D}_{\infty}, \mathcal{C}_{\infty}, \mathcal{F}_{\infty}$ obtaining the same result.

The points $\mathcal{C}_{\infty}$ and $\mathcal{F}_{\infty}$ represent a state in which the total energy density of the curvature fluid dominates and corresponds to closed Einstein universes. For these points $x$ and $y$ goes to infinity at the same rate $(\theta=\pi / 4)$. In this limit, the system (19) reduces to the equation

$$
x^{\prime}=\frac{1-2 n}{n-1} x^{2}
$$


Table 3. Asymptotic fixed points, $\theta$ coordinates and solutions for $R^{n}$-gravity in vacuum.

\begin{tabular}{ccc}
\hline Point & $\theta$ & Behavior \\
\hline $\mathcal{A}_{\infty}$ & 0 & $\left|\mathcal{N}-\mathcal{N}_{\infty}\right|=\left[C_{1} \pm \frac{C_{0}}{2}\left(t-t_{0}\right)\right]^{2}$ \\
$\mathcal{B}_{\infty}$ & $\pi / 4$ & $\left|\mathcal{N}-\mathcal{N}_{\infty}\right|=\left[C_{1} \pm C_{0}\left|\frac{n-1}{2 n-1}\right|\left(t-t_{0}\right)\right]^{\frac{2 n-1}{n-1}}$ \\
$\mathcal{C}_{\infty}$ & $\pi / 2$ & $\left|\mathcal{N}-\mathcal{N}_{\infty}\right|=\left[C_{1} \pm \frac{C_{0}}{2}\left(t-t_{0}\right)\right]^{2}$ \\
$\mathcal{D}_{\infty}$ & $\pi$ & $\left|\mathcal{N}-\mathcal{N}_{\infty}\right|=\left[C_{1} \pm \frac{C_{0}}{2}\left(t-t_{0}\right)\right]^{2}$ \\
$\mathcal{E}_{\infty}$ & $5 \pi / 4$ & $\left|\mathcal{N}-\mathcal{N}_{\infty}\right|=\left[C_{1} \pm C_{0}\left|\frac{n-1}{2 n-1}\right|\left(t-t_{0}\right)\right]^{\frac{2 n-1}{n-1}}$ \\
$\mathcal{F}_{\infty}$ & $3 \pi / 2$ & $\left|\mathcal{N}-\mathcal{N}_{\infty}\right|=\left[C_{1} \pm \frac{C_{0}}{2}\left(t-t_{0}\right)\right]^{2}$ \\
\hline
\end{tabular}

which admits the solution

$$
x=\frac{n-1}{(2 n-1)\left(\mathcal{N}-\mathcal{N}_{\infty}\right)},
$$

where $\mathcal{N}$, is an integration constant. In the same way, the (23) can be written as

$$
\frac{H^{\prime}}{H}=\frac{n x}{n-1} .
$$

Substituting (41) in (42) and integrating we obtain

$$
|H|=|\dot{\mathcal{N}}|=C_{0}\left|\mathcal{N}-\mathcal{N}_{\infty}\right|^{\frac{n}{2 n-1}},
$$

where $C_{0}$ is a positive integration constant. Integrating again with respect to the variable $t$ we obtain

$$
\left|\mathcal{N}-\mathcal{N}_{\infty}\right|=\left[C_{1} \pm C_{0}\left|\frac{n-1}{2 n-1}\right|\left(t-t_{0}\right)\right]^{\frac{2 n-1}{n-1}} / ; .
$$

This solution represents an expansion if $n<1 / 2$ and $n>1$, a contraction if $1 / 2<n<1$.

The value of $n$ influences also the stability of these fixed points (see Table 4). In particular, we see that only the points $\mathcal{B}_{\infty}, \mathcal{D}_{\infty}, \mathcal{F}_{\infty}$ can be attractors. For the values of $n$ suggested by the observations only the points $\mathcal{D}_{\infty}$ or $\mathcal{F}_{\infty}$ are attractors and we can conclude by saying that, for these values of $n$, the evolution is always given by (44).

Summarizing the above results, we can say that the global behavior of vacuum $R^{n}$ gravity exhibits transient Milne and power law states that eventually approach the solutions $\mathcal{C}, \mathcal{D}$ or $\mathcal{B}_{\infty}, \mathcal{D}_{\infty}, \mathcal{F}_{\infty}$ depending on the value of the parameter $n$ and the initial conditions. For $n$ far from one, only the points $\mathcal{C}$ and $\mathcal{D}_{\infty}, \mathcal{F}_{\infty}$ are attractors, the first one representing an accelerating solution. In the neighborhood of $n=1$ the physics becomes more complex because $\mathcal{D}$ and $\mathcal{B}_{\infty}$ can acquire a stable character (node or focus) and $\mathcal{C}$ changes its stability. Finally, the phase space analysis shows that, for these type of theories, the sign of the curvature remains fixed once it has been set by the initial conditions. This is an important aspect of dynamics of the vacuum 
Table 4. Stability of the asymptotic fixed points for $R^{n}$-gravity in vacuum.

\begin{tabular}{cccc}
\hline Point & $n<1 / 2$ & $1 / 2<n<1$ & $n>1$ \\
\hline $\mathcal{A}_{\infty}$ & repulsive & repulsive & repulsive \\
$\mathcal{B}_{\infty}$ & saddle & attractive & saddle \\
$\mathcal{C}_{\infty}$ & saddle & saddle & repulsive \\
$\mathcal{D}_{\infty}$ & attractive & attractive & attractive \\
$\mathcal{E}_{\infty}$ & saddle & repulsive & saddle \\
$\mathcal{F}_{\infty}$ & saddle & saddle & attractive \\
\hline
\end{tabular}

model because, if the coordinate $y$ of the point $\mathcal{C}$ is different from the sign of the initial condition for the curvature, the solution $\mathcal{C}$ is never reached. This means that $\mathcal{C}$ cannot be considered a global attractor for the vacuum case and an accelerating expansion phase is not guaranteed.

\section{The Matter Case}

When matter is present (as a perfect fluid), each of the cosmological equations gain a new source term containing the energy density. This modification has deep consequences because, as we can see from (14][15), the matter terms are coupled with a generic power of the curvature. Now, since in general the sign of the Ricci scalar is not fixed, these terms will not be defined for every real value of $n$. In particular we can allow only values of $n$ belonging to the set of the relative numbers $\mathcal{Z}$ and the subset of the rational numbers $\mathcal{Q}$ which can be expressed as fractions with an odd denominator

In this sense, the presence of matter induces a natural constraint through the field equations on $R^{n}$-gravity. In other words, if we suppose the field equations (77) to be valid, not all the higher order theories of gravity are consistent in presence of standard matter. This difference between vacuum and non-vacuum physics is not present in GR but it is a common feature of higher order theories.

The constraints on $n$ also introduces technical problems because some of the results that we will present below require numerical calculations. This means that the result should be expressed in terms of the allowed set of values of $n$, but this is neither an easy nor a useful task. For this reason, we will work as if $n$ was unconstrained, supposing that all the intervals that we devise are meant to represent the subset of allowed values of $n$ within these intervals, and that their boundaries approximate the nearest allowed value of $n$.

With this in mind, let us now derive the dynamics for the matter case. We again 
start with (15), and define the expansion normalized variables

$$
\begin{aligned}
& x=\frac{\dot{R}}{R H}(n-1), \\
& y=\frac{R}{6 n H^{2}}(1-n), \\
& z=\frac{\rho}{3 n \chi(n) H^{2} R^{n-1}}, \\
& K=\frac{\kappa}{a^{2} H^{2}},
\end{aligned}
$$

where $z$ represents the contribution of standard matter in the form of the energy density weighted by a power of the Ricci curvature. In order to write down a dynamical equation for this variable, we need an expression for the time derivative of the energy density. Such

an expression can be obtained from the Bianchi Identities $T_{\mu \nu}^{T O T} ; \nu=0$ which describe the conservation properties. As we have seen, if the gravitational field equations (77) hold, it is possible to show 25, 24 that by virtue of the Bianchi identities, whatever the form of the gravitational Lagrangian, the conservation equation for matter are simply given by (17).

Differentiating (45) with respect to the logarithmic time, we obtain the autonomous system

$$
\begin{aligned}
x^{\prime} & =x-2 x^{2}+\frac{(2+n x) y}{1-n}-(2+x) K+2-z(1+3 w), \\
y^{\prime} & =\frac{x y}{n-1}+2 y\left(\frac{y n}{1-n}+2+K\right), \\
z^{\prime} & =-3 z(1+w)+2 z\left(2+\frac{y n}{n-1}+K\right)-x z, \\
K^{\prime} & =2 K\left(1+\frac{y n}{n-1}+K\right),
\end{aligned}
$$

with the constraint

$$
1+x-y-z+K=0 .
$$

As before, we can reduce the number of variables by substituting (150) into the other equations and recognizing that the spatial curvature equation is a linear combination of the other three. In this way, the final system becomes

$$
\begin{aligned}
& x^{\prime}=\frac{2(n-2) y}{n-1}-2 x-2 x^{2}-\frac{x y}{n-1}+(1+x) z-3 z w, \\
& y^{\prime}=\frac{y}{n-1}[(3-2 n) x-2 y+2(n-1) z+2(n-1)], \\
& z^{\prime}=z\left(2 z-1-3 x-\frac{2 y}{n-1}-3 w\right) .
\end{aligned}
$$

Note that, as in the previous system, $y=0 \Rightarrow y^{\prime}=0$, but now also $z=0 \Rightarrow z^{\prime}=0$ holds. Consequently the two planes $y=0$ and $z=0$ correspond to two invariant submanifolds. Since for $z=0$ (51) reduces to (21), we would be tempted to interpret the plane $z=0$ to be the vacuum invariant submanifold of the phase space. This is not 
entirely correct. In order to understand this point, let us write the energy density $\rho$ in terms of the variables (45). We have

$$
\rho=z y^{n-1} H^{2 n} .
$$

Form this formula it is clear that when $z=0($ and $y \neq 0)$ the density is zero whatever the value of the parameter $n$. Instead if $y=0$ (and $z \neq 0$ ) the behavior depends on the value of $n$. In particular, the density is zero for $y=0$ and $n>1$ and divergent for $n<1$. If both $x$ and $z$ are equal to zero for $n<1$ the behavior of $\rho$ is undetermined and can be found only by direct substitution in the cosmological equations.

As a consequence, for $n>1$ both $(x, y)$ and the $(x, z)$ planes are invariant vacuum manifolds, but if $n<1$ the vacuum submanifold is not necessarily compact because it is made by $(x, y)$ and other (in principle) submanifolds of the $(x, z)$ plane.

The fact that $z=0$ and $y=0$ are invariant submanifolds also suggests that there are no orbits which can cross these planes: if we choose initial conditions $y_{i}=0\left(z_{i}=0\right)$, the equations (51) imply that the orbits will never leave the plane $y=0(z=0)$. As a consequence, if the evolution has initial conditions for which $y_{i} \neq 0\left(z_{i} \neq 0\right)$ they can approach the plane $y=0$ only asymptotically. Consequently, like in the vacuum case, no global attractor exists in these models because no orbit can, in general, cover all of the phase space.

\subsection{Finite Analysis}

Setting $x^{\prime}=0, y^{\prime}=0, z^{\prime}=0$ and solving for $x, y, z$ we obtain the fixed points:

$$
\begin{array}{ll}
\mathcal{A}=\{y \rightarrow 0, x \rightarrow 0, z \rightarrow 0\}, & K=-1, \\
\mathcal{B}=\{y \rightarrow 0, x \rightarrow-1, z \rightarrow 0\}, & K=0, \\
\mathcal{C}=\left\{y \rightarrow \frac{4 n-5}{2 n-1}, x \rightarrow \frac{2(n-2)}{2 n-1}, z \rightarrow 0\right\}, & K=0, \\
\mathcal{D}=\left\{y \rightarrow 2(n-1)^{2}, x \rightarrow-2(n-1), z \rightarrow 0\right\}, & K=2 n^{2}-2 n-1, \\
\mathcal{E}=\{y \rightarrow 0, x \rightarrow-1-3 w, z \rightarrow-1-3 w\}, & K=-1, \\
\mathcal{F}=\{y \rightarrow 0, x \rightarrow 1-3 w, z \rightarrow 2-3 w\}, & K=0, \\
\mathcal{G}=\left\{y \rightarrow \frac{(n-1)(4 n-12 w-3)}{2 n^{2}}, x \rightarrow-\frac{3(n-1)(1+w)}{n},\right. &
\end{array}
$$

Note that the fixed points $\mathcal{A}, \mathcal{B}, \mathcal{C}, \mathcal{D}$ have the same coordinates (22) found in the vacuum case, with the additional condition that the variable $z$ has to be zero.

Also in this case we have two different classes of fixed points, one independent of the value of the parameters $n(\mathcal{A}, \mathcal{B}, \mathcal{E}, \mathcal{F})$ and one that changes its coordinates with $n$ $(\mathcal{C}, \mathcal{D}, \mathcal{G})$.

There are also values of $n$ for which two of the fixed points $(\mathcal{C}, \mathcal{G})$ acquire an asymptotic character, namely $0,1 / 2$. However, $n=1 / 2$ is not an allowed value in this 
Table 5. Coordinates of the fixed points and solutions for $R^{n}$-gravity with matter.

\begin{tabular}{|c|c|c|}
\hline Point & Coordinates $(x, y, z)$ & Scale Factor \\
\hline $\mathcal{A}$ & {$[0,0,0]$} & $a=a_{0}\left(t-t_{0}\right)$ \\
\hline $\mathcal{B}$ & {$[-1,0,0]$} & $a=a_{0}\left(t-t_{0}\right)^{1 / 2}($ only for $n=3 / 2)$ \\
\hline $\mathcal{C}$ & {$\left[\frac{2(n-2)}{2 n-1}, \frac{4 n-5}{2 n-1}, 0\right]$} & $a=a_{0} t^{\frac{(1-n)(2 n-1)}{n-2}}$ \\
\hline $\mathcal{D}$ & {$\left[2(1-n), 2(n-1)^{2}, 0\right]$} & $\left\{\begin{array}{ccc}a=\frac{k t}{2 n^{2}-2 n-1} & \text { if } & k \neq 0 \\
a=a_{0} t & \text { if } & k=0\end{array}\right.$ \\
\hline $\mathcal{E}$ & {$[-1-3 w, 0,-1-3 w]$} & $a=a_{0}\left(t-t_{0}\right)$ \\
\hline $\mathcal{F}$ & {$[1-3 w, 0,2-3 w]$} & $a=a_{0}\left(t-t_{0}\right)^{1 / 2}($ only for $n=3 / 2)$ \\
\hline $\mathcal{G}$ & $\begin{array}{c}{\left[-\frac{3(n-1)(1+w)}{n}, \frac{(n-1)[4 n-3(w+3)]}{2 n^{2}},\right.} \\
\left.\frac{n(13+9 w)-2 n^{2}(4+3 w)-3(1+w)}{2 n^{2}}\right]\end{array}$ & $a=a_{0} t^{\frac{2 n}{3(1+w)}}$ \\
\hline
\end{tabular}

\begin{tabular}{cc}
\hline Point & Matter Density \\
\hline $\mathcal{A}$ & $\rho=0$ \\
$\mathcal{B}$ & $\rho=0$ \\
$\mathcal{C}$ & $\rho=0$ \\
$\mathcal{D}$ & $\rho=0$ \\
$\mathcal{E}$ & $\rho=0$ for $n>1$ or $\rho$ divergent for $n<1$ \\
$\mathcal{F}$ & $\rho=0$ \\
$\mathcal{G}$ & $\rho=\rho_{0} t^{-2 n}$ \\
& $(4 n-3(1+w))^{n-1}\left[2 n^{2}(4+3 w)-n(13+9 w)+3(1+w)\right]$ \\
\hline
\end{tabular}

case and $n=0$ corresponds to a constant Lagrangian for the gravitational interaction. These values of $n$ are not interesting for our purposes and are therefore excluded.

Merging occurs for $w$ in the Zel'dovic interval, excluding $n=1$, for $\mathcal{A}$ and $\mathcal{B}$ at $n=5 / 4$, for $\mathcal{C}$ and $\mathcal{D}$ at $n=\frac{1}{2}(1+\sqrt{3})$ and $n=\frac{1}{2}(1-\sqrt{3})$, for $\mathcal{C}$ and $\mathcal{G}$ when $n$ is solution of the equation $(8+6 w) n^{2}-(13+9 w) n+3(1+w)=0$. None of this values are allowed in the presence of matter, so we can consider these fixed points to be always distinct.

The stability of the fixed points can be obtained, as usual, by linearizing the system (51) and are summarized in Tables (7) 8) and 9). Because the phase space is three dimensional, the general dynamical behavior is not easy to visualize. We therefore do not give any graphical representation as in the vacuum case and focus only on the stability of the fixed points.

The fixed point $\mathcal{A}$ has always a saddle-node character because the eigenvalues of 
Table 6. Eigenvalues of the fixed points for $R^{n}$-gravity with matter.

\begin{tabular}{cc}
\hline Point & Eigenvalues \\
\hline $\mathcal{A}$ & $(-2,2,-1-3 w)$ \\
$\mathcal{B}$ & $\left(2, \frac{4 n-5}{n-1}, 2-3 w\right)$ \\
$\mathcal{C}$ & $\left(\frac{4 n-5}{n-1}, \frac{2+4 n-4 n^{2}}{1-3 n+2 n^{2}}, \frac{-3-13 n-8 n^{2}-3 w+9 n w-6 n^{2} w}{(n-1)(2 n-1)}\right)$ \\
$\mathcal{D}$ & $(-2+n-\sqrt{3 n(3 n-4)},-2+n+\sqrt{3 n(3 n-4)}, 2 n-3(1+w))$ \\
$\mathcal{E}$ & $\left(-2, \frac{2 n-3-3 w}{n-1}, 1+3 w\right)$ \\
$\mathcal{F}$ & $\left(2, \frac{4 n-3-3 w}{n-1}, 3 w-2\right)$ \\
$\mathcal{G}$ & $\left(\frac{3-2 n+3 w}{n}, \frac{P_{1}(n, w)-\sqrt{P_{2}(n, w)}}{4 n(n-1)}, \frac{P_{1}(n, w)+\sqrt{P_{2}(n, w)}}{4 n(n-1)}\right)$, \\
& $P_{1}(n, w)=(3(1+w)+3 n((2 n-3) w-1))$ \\
& $P_{2}(n, w)=(n-1)\left[4 n^{3}(8+3 w)^{2}-4 n^{2}(152+3 w(55+18 w))\right.$ \\
& $\left.+3 n(1+w)(139+87 w)-81(1+w)^{2}\right]$ \\
\hline
\end{tabular}

Table 7. Stability of the fixed points for $R^{n}$-gravity with matter (dust or radiation). The term "spiral" has been used for pure attractive focus-nodes, the term "anti-spiral" for pure repulsive focus-nodes, and the term "saddle-focus" for unstable focus-nodes. The point $\mathcal{G}$ has been treated separately because of the approximations used.

\begin{tabular}{ccccc}
\hline & $n<\frac{1}{2}(1-\sqrt{3})$ & $\frac{1}{2}(1-\sqrt{3})<n<0$ & $0<n<1 / 2$ & $1 / 2<n<1$ \\
\hline $\mathcal{A}$ & saddle & saddle & saddle & saddle \\
$\mathcal{B}$ & repellor & repellor & repellor & repellor \\
$\mathcal{C}$ & attractor & saddle & saddle & attractor \\
$\mathcal{D}$ & saddle & attractor & spiral & spiral \\
$\mathcal{E}$ & saddle & saddle & saddle & saddle \\
$\mathcal{F}$ & saddle & saddle & saddle & saddle \\
\hline
\end{tabular}

\begin{tabular}{ccccc}
\hline & $1<n<5 / 4$ & $5 / 4<n<4 / 3$ & $4 / 3<n<\frac{1}{2}(1+\sqrt{3})$ & $n>\frac{1}{2}(1+\sqrt{3})$ \\
\hline $\mathcal{A}$ & saddle & saddle & saddle & saddle \\
$\mathcal{B}$ & saddle & repellor & repellor & repellor \\
$\mathcal{C}$ & repellor & saddle & saddle & attractor \\
$\mathcal{D}$ & spiral & spiral & attractor & saddle \\
$\mathcal{E}$ & saddle & saddle & saddle & saddle \\
$\mathcal{F}$ & saddle & saddle & saddle & saddle \\
\hline
\end{tabular}


Table 8. Stability of the fixed points for $R^{n}$-gravity with stiff matter. The term "spiral" has been used for pure attractive focus-nodes, the term "anti-spiral" for pure repulsive focus-nodes, and the term "saddle-focus" for unstable focus-nodes. The point $\mathcal{G}$ has been treated separately because of the approximations used.

\begin{tabular}{cccccc}
\hline & $n<\frac{1}{2}(1-\sqrt{3})$ & $\frac{1}{2}(1-\sqrt{3})<n<0$ & $0<n<1 / 2$ & $1 / 2<n<1$ & $1<n<\frac{1}{14}(11+\sqrt{37})$ \\
\hline $\mathcal{A}$ & saddle & saddle & saddle & saddle & saddle \\
$\mathcal{B}$ & saddle & saddle & saddle & saddle & saddle \\
$\mathcal{C}$ & attractor & saddle & saddle & attractor & repellor \\
$\mathcal{D}$ & saddle & attractor & spiral & spiral & spiral \\
$\mathcal{E}$ & saddle & saddle & saddle & saddle & saddle \\
$\mathcal{F}$ & saddle & saddle & saddle & saddle & saddle \\
\hline
\end{tabular}

\begin{tabular}{ccccc}
\hline & $\frac{1}{14}(11+\sqrt{37})<n<4 / 3$ & $4 / 3<n<\frac{1}{2}(1+\sqrt{3})$ & $\frac{1}{2}(1+\sqrt{3})<n<3 / 2$ & $n>3 / 2$ \\
\hline $\mathcal{A}$ & saddle & saddle & saddle & saddle \\
$\mathcal{B}$ & saddle & saddle & saddle & saddle \\
$\mathcal{C}$ & saddle & saddle & attractor & attractor \\
$\mathcal{D}$ & spiral & attractor & saddle & saddle \\
$\mathcal{E}$ & saddle & saddle & saddle & saddle \\
$\mathcal{F}$ & saddle & saddle & saddle & repellor \\
\hline
\end{tabular}

the linearized matrix do not depend on $n$ and have an alternate sign whatever the value of $w$.

The fixed point $\mathcal{B}$ is an unstable node for $n<1$ and $n>5 / 4$ if the standard matter is dust or radiation and a saddle otherwise. In the stiff matter case this point is always a saddle.

The fixed point $\mathcal{C}$ behaves like a pure unstable node for $1<n<5 / 4$ if the standard matter is dust or radiation and for $1<n<\frac{1}{14}(11+\sqrt{37})$ in case of stiff matter. A pure stable character is instead found for $n<\frac{1}{2}(1-\sqrt{3}), 1 / 2<n<1$ and $n>\frac{1}{2}(1+\sqrt{3})$, whatever the value of the parameter $w$. For all the other values of $n$ this point is a saddle-node.

The fixed point $\mathcal{D}$ has eigenvalues that involve a square root of a function of $n$. This means that when this function is negative, the eigenvalues are complex and these points behave like focus-nodes. In particular, for $n<0$ and $n>4 / 3$ this point is a pure attractive node if $\frac{1}{2}(1-\sqrt{3})<n \leqslant 0$ and $4 / 3 \leqslant n<\frac{1}{2}(1+\sqrt{3})$ or a saddle-node otherwise. For $0<n<4 / 3$ the eigenvalues are complex and an analysis of the real parts show that the focus-nodes are always attractive.

The fixed point $\mathcal{E}$ is a saddle-node whatever the values of $n$ and $w$.

The fixed point $\mathcal{F}$ is a saddle-node for every value of $n$ if the matter is dust or 
Table 9. Stability of the fixed point $\mathcal{G}$. The term "spiral" has been used for pure attractive focus-nodes, the term "anti-spiral" for pure repulsive focus-nodes, and the term "saddle-focus" for unstable focus-nodes.

\begin{tabular}{cccccc}
\hline & $n \lesssim 0.33$ & $0.33 \lesssim n \lesssim 0.35$ & $0.35 \lesssim n \lesssim 0.37$ & $0.37 \lesssim n \lesssim 0.71$ & $0.71 \lesssim n \lesssim 1$ \\
\hline$w=0$ & saddle & saddle-focus & saddle-focus & saddle-focus & saddle \\
$w=1 / 3$ & saddle & saddle & saddle-focus & saddle-focus & saddle-focus \\
$w=1$ & saddle & saddle & saddle & saddle-focus & saddle-focus \\
\hline
\end{tabular}

\begin{tabular}{ccccc}
\hline & $1 \lesssim n \lesssim 1.220$ & $1.220 \lesssim n \lesssim 1.223$ & $1.223 \lesssim n \lesssim 1.224$ & $1.224 \lesssim n \lesssim 1.28$ \\
\hline$w=0$ & saddle-focus & saddle-focus & saddle-focus & saddle-focus \\
$w=1 / 3$ & saddle-focus & saddle-focus & saddle-focus & saddle-focus \\
$w=1$ & saddle & repellor & saddle & anti-spiral \\
\hline
\end{tabular}

\begin{tabular}{ccccc}
\hline & $1.28 \lesssim n \lesssim 1.32$ & $1.32 \lesssim n \lesssim 1.47$ & $1.47 \lesssim n \lesssim 1.50$ & $n \gtrsim 1.50$ \\
\hline$w=0$ & saddle-focus & saddle & saddle & saddle \\
$w=1 / 3$ & saddle & saddle & saddle & saddle \\
$w=1$ & anti-spiral & anti-spiral & repellor & saddle \\
\hline
\end{tabular}

radiation. In the stiff matter case, this fixed point is an unstable node if $n>\frac{3}{2}$.

The calculations of the eigenvalues of the fixed point $\mathcal{G}$ cannot be performed in an exact way for a general $n$ because they involve the solution of complete algebraic equations of order higher than two. Numerical calculations show that there are complex eigenvalues for $0.33 \lesssim n \lesssim 0.71$ and $1 \lesssim n \lesssim 1.33$ if $w=0,0.35 \lesssim n \lesssim 1.28$ if $w=1 / 3$, $0.37 \lesssim n \lesssim 1$ and $1.224 \lesssim n \lesssim 1.47$, if $w=1$. For all these values of $n, \mathcal{G}$ behaves like a saddle focus. For the other values of $n$ this point is always a saddle-node apart in case of stiff matter, for which $1.220 \lesssim n \lesssim 1.223$ and $1.47 \lesssim n \lesssim 1.5$. In this case we have a pure repulsive node $\boldsymbol{\top}$.

Using the same procedure of the vacuum case, the coordinates of the fixed points (53) can be used to find the behavior of the scale factor. The equation (231) generalizes to

$$
\dot{H}=\left(x_{C}+\frac{y_{C}}{n-1}-z_{C}-1\right) H^{2} .
$$

If $n \neq 1$ and

$$
(n-1)\left(x_{C}-z_{C}-1\right)-y_{C} \neq 0
$$

I Also in this case we will not investigate the behavior of this model in the bifurcations leaving this task for a future paper. 
this equation can be integrated to give

$$
a=a_{0}\left(t-t_{0}\right)^{\alpha} \quad \text { with } \quad \alpha=\left(1-x_{C}-\frac{y_{C}}{n-1}+z_{C}\right)^{-1} .
$$

However, the characterization of the solution in this case requires also the derivation of the energy density $\rho$ which is given by the (52).

Since for $z_{C}=0$ the solution (56) reduces to the (25), the fixed points $\mathcal{A}, \mathcal{B}, \mathcal{C}, \mathcal{D}$ are associated with the same solutions given in the vacuum case. The points $\mathcal{C}$ and $\mathcal{D}$ have $y \neq 0$ and represent vacuum solution for every value of $n$ and using the cosmological equations is it possible to show that this is also true for the solution associated with $\mathcal{A}$ and $\mathcal{B}$.

The equation (56) also allows one to find a solution for $\mathcal{E}$. This point correspond to a vacuum Milne solution, but since the energy density in this point is divergent for $n<1$ we consider this point physical only for $n>1$.

The solution for point $\mathcal{F}$, instead, cannot be found via the (56) because the condition (55) is violated. A direct resolution of (154) tells us that this point represents a solution only if $\rho=0$ and $n=3 / 2$, but this value of the parameter is forbidden in this case.

The fixed point $\mathcal{G}$ is instead a new solution with respect to the vacuum case. The scale factor and the Hubble parameter, are in this case,

$$
a=a_{0}\left(t-t_{0}\right)^{\frac{2 n}{3(1+w)}}
$$

and the energy density is given by

$$
\rho=\rho_{0} t^{-2 n}
$$

with

$$
\begin{aligned}
\rho_{0}= & (-1)^{n} \chi(n) 3^{-n} 2^{2 n-1} n^{n}(1+w)^{-2 n} \times \\
& (4 n-3(1+w))^{n-1}\left[2 n^{2}(4+3 w)-n(13+9 w)+3(1+w)\right] .
\end{aligned}
$$

Thus $\mathcal{G}$ represents a power law regime which, for $n>0$, is an expanding solution with $\rho$ decreasing in time, and for $n<0$ a contracting solution with $\rho$ increasing in time. If $n$ is close to 1 , this solution can be thought of as an "almost-Friedman" solution. Instead, for $n>\frac{3}{2}(1+w)$ this solution corresponds to accelerating expansion.

Unfortunately, since $\rho_{0}$ is a function of $n$ and $w, \rho$ is not necessarily positive and consequently $\mathcal{G}$ is not necessarily a physical point. The values of $n$ for which $\mathcal{G}$ is physical can be found by solving the inequality $\rho_{0}>0$. We can distinguish two different cases: $n$ belonging to the set of even integers or allowed rational numbers with an even numerator, which we call $\mathcal{N}_{\text {even }}$, and $n$ belonging to the odd integers or allowed rationals with odd numerator, which we call $\mathcal{N}_{\text {odd }}$.

$\left(n \in \mathcal{N}_{\text {even }}\right)$ In this case we require that

$$
(4 n-3(1+w))\left[2 n^{2}(4+3 w)-n(13+9 w)+3(1+w)\right]>0 .
$$


This inequality is satisfied for $0.28 \lesssim n \lesssim 0.75$ and for $n \gtrsim 1.35$ if $w=0$, for $0.31 \lesssim n \lesssim 1$ and $n \gtrsim 1.29$ if $w=1 / 3$, for $0.35 \lesssim n \lesssim 1.22$ and $n \gtrsim 1.5$ if $w=1$. If $n<1$ we should also add the constraint $(4 n-3(1+w)) \neq 0$ to avoid divergences. However, the solution of this equation for physical choices of $w$ have values of $n$ which are not forbidden by the above inequalities, or do not correspond to the case $n=1$. As a consequence, the matter energy density always remains finite.

$\left(n \in \mathcal{N}_{\text {odd }}\right)$ This case can be split in two different subcases

$n>0$ for which

$$
\left[2 n^{2}(4+3 w)-n(13+9 w)+3(1+w)\right]<0,
$$

which leads to $0.28 \lesssim n \lesssim 1.35$ if $w=0,0.31 \lesssim n \lesssim 1.29$ if $w=1 / 3$, $0.35 \lesssim n \lesssim 1.22$ if $w=1$, the value $n=1$ being excluded.

$n<0$ for which

$$
\left[2 n^{2}(4+3 w)-n(13+9 w)+3(1+w)\right]>0,
$$

leads to $n \lesssim 0$ for $w=0,1 / 3,1$.

It should be also pointed out that for $n=\frac{13+9 w \pm \sqrt{73+66 w+9 w^{2}}}{2(8+6 w)}$, the density is zero. However these values are not allowed in the matter case, so the matter density is non-zero for all physical values of $n$.

Using (52), the conditions above can be also read as conditions on the coordinates of $\mathrm{G}$. In particular, the point $\mathrm{G}$ will represent a physical point only if

$$
\begin{aligned}
& z y>0 \quad \text { for } \quad n \in \mathcal{N}_{\text {even }} \text {; } \\
& \left\{\begin{array}{ll}
z<0, & n>0 \\
z>0, & n<0
\end{array} \quad \text { for } \quad n \in \mathcal{N}_{\text {odd }} .\right.
\end{aligned}
$$

Again, it is useful to define the deceleration parameter $q$ and obtain the regions of the phase space in which correspond to accelerated evolution. We have

$$
q=-\frac{H^{\prime}}{H^{2}}-1=z-x-\frac{y}{n-1}
$$

which means, for $q>0$,

$$
\left\{\begin{array} { l } 
{ n > 1 } \\
{ ( z - x ) ( n - 1 ) - y > 0 }
\end{array} \left\{\begin{array}{l}
n<1 \\
(z-x)(n-1)-y<0
\end{array}\right.\right.
$$

Thus, chosen $n$, the phase space is divided in two regions which correspond to accelerating and decelerating evolution. It is easy to check that the conditions (63) are never satisfied for the points $\mathcal{A}, \mathcal{B}, \mathcal{D}$ and for $\mathcal{E}, \mathcal{F}$ (as we would expect from the solutions given above). For the point $\mathcal{C}$ the constraint on $n$ are the same as (33) and for the point $\mathcal{G}$ we have an accelerated evolution only if $n>\frac{1}{2}(3+3 \omega)$ (expansion) and $n<0$ (contraction). 


\subsection{Asymptotic analysis}

As already discussed for the vacuum case, we complete the phase space analysis by investigating the asymptotic regime. The compactification of the phase space is achieved in the same way as the vacuum case, the difference being that now we now use spherical polar coordinates $(r, \theta, \phi)$ for which

$$
x \rightarrow r \sin (\theta) \cos (\phi), \quad y \rightarrow r \sin (\theta) \sin (\phi), \quad z \rightarrow r \cos (\theta),
$$

where $r \in[0, \infty[, \theta \in[0, \pi], \phi \in[0,2 \pi]$. As in the vacuum case, the actual compactification is achieved transforming the radial coordinate $r$ in $\mathcal{R}$. In the limit $\mathcal{R} \rightarrow 1$ the dynamic equations (51) become

$$
\begin{aligned}
& \mathcal{R}^{\prime} \rightarrow \frac{1}{4(n-1)}\left\{8(n-1) \cos ^{3}(\theta)-2(n-1) \cos (\theta) \sin ^{2}(\theta)(\cos (2 \theta)-3)-4 \cos ^{2}(\theta) \sin (\theta) \times\right. \\
& {[3(n-1) \cos (\phi)+2 \sin (\phi)]+\sin ^{3}(\theta)[(9-8 n) \cos (\phi)-\cos (3 \phi)-7 \sin (\phi)} \\
& +\sin (3 \phi)]\} \\
& \mathcal{R} \theta^{\prime} \rightarrow \frac{\cos (\phi) \sin (2 \theta)\{2 n \sin (\theta)+2 \cos (\phi)[(n-1) \cos (\theta)+\sin (\theta)(\sin (\phi)-\cos (\phi))]\}}{4(n-1)(1-\mathcal{R})}(66) \\
& \mathcal{R} \phi^{\prime} \rightarrow \frac{1}{(n-1)(1-\mathcal{R})}\{\cos (\phi) \sin (\phi)[(n-1) \cos (\theta)+\sin (\theta)(\sin (\phi)-\cos (\phi))]\} .
\end{aligned}
$$

This system has many interesting features. First of all, the above equations are parameterized by $n$ but not by the barotropic factor $w$. This means that the asymptotic regime is independent of the nature of the perfect fluid that we choose. In addition, since there is no $\mathcal{R}$ dependence in the (65), the fixed points are determined only by the angular equations. Therefore, in order to find the asymptotic fixed points, we can neglect the $\mathcal{R}^{\prime}$ equation and deal only with the $\left(\theta^{\prime}, \phi^{\prime}\right)$ system.

Looking carefully at the system (66,67) we realize that it presents a non-trivial structure because the polynomials in the R.H.S are not prime to each other. This means that we have an infinite number of fixed points due to the zeros of the common factor. These points will define "fixed subspaces" of the total phase space in which every single point has, in principle, its own stability.

Setting $\theta^{\prime}=0$ and $\phi^{\prime}=0$, we obtain the coordinates of the fixed points. Although we obtain 26 independent solutions, many of them are associated with the same fixed point on the sphere due to the periodic nature of the trigonometric functions in 6667) and the peculiar topology of the sphere. The results are summarized in Table 10 ,

We can distinguish a first set of eight ordinary fixed points whose coordinates do not depend on the value of $n$ and are therefore common to all the $R^{n}$ models, two "fixed lines" $L 1$ and $L 2$ again independent of $n$ and other four fixed points on $L 1$ and $L 2$ whose coordinates depend on $n$.

The solutions associated with the first four $\left(\mathcal{A}_{\infty}-\mathcal{D}_{\infty}\right)$ can be obtained using the same kind of procedure used in the vacuum case, we can obtain their solutions (see Table [11). 
Table 10. Coordinates of the asymptotic fixed points and relative growing rates in non-vacuum $R^{n}$-gravity. Here "ind." represents an indeterminate form.

\begin{tabular}{cclccc}
\hline & & & \multicolumn{3}{c}{ Growth Rates } \\
\cline { 5 - 6 } Point & $(\theta, \phi)$ & Coordinates & $y / x$ & $z / x$ & $y / z$ \\
\hline $\mathcal{A}_{\infty}$ & $(0,0)$ & $x, y \rightarrow 0, z \rightarrow+\infty$ & 0 & $\infty$ & 0 \\
$\mathcal{B}_{\infty}$ & $(\pi, \pi)$ & $x, y \rightarrow 0, z \rightarrow-\infty$ & 0 & $\infty$ & 0 \\
$\mathcal{C}_{\infty}$ & $(\pi / 2,0)$ & $y, z \rightarrow 0, x \rightarrow+\infty$ & 0 & 1 & ind. \\
$\mathcal{D}_{\infty}$ & $(-\pi / 2, \pi)$ & $y, z \rightarrow 0, x \rightarrow-\infty$ & 0 & 1 & ind. \\
$\mathcal{E}_{\infty}$ & $(\pi / 4,0)$ & $x, z \rightarrow+\infty, y \rightarrow 0$ & 0 & 1 & 0 \\
$\mathcal{F}_{\infty}$ & $(3 \pi / 4, \pi)$ & $x, z \rightarrow-\infty, y \rightarrow 0$ & 0 & 1 & 0 \\
$\mathcal{G}_{\infty}$ & $(\pi / 2, \pi / 4)$ & $x, y \rightarrow+\infty, z \rightarrow 0$ & 1 & 0 & $\infty$ \\
$\mathcal{H}_{\infty}$ & $(\pi / 2,5 \pi / 4)$ & $x, y \rightarrow-\infty, z \rightarrow 0$ & 1 & 0 & $\infty$ \\
\hline Line $L 1$ & $(\theta, \pi / 2)$ & $y, z \rightarrow+\infty, x \rightarrow 0$ & $\infty$ & $\infty$ & $\tan (\theta)$ \\
Line $L 2$ & $(\theta, 3 \pi / 2)$ & $y, z \rightarrow-\infty, x \rightarrow 0$ & $\infty$ & $\infty$ & $\tan (\theta)$ \\
\hline $\mathcal{I}_{\infty}^{1}$ & $(\arctan (n-1), \pi / 2)$ & $y, z \rightarrow+\infty, x \rightarrow 0$ & $\infty$ & $\infty$ & $n-1$ \\
$\mathcal{I}_{\infty}^{2}$ & $(-\arctan (n-1), \pi / 2)$ & $y, z \rightarrow+\infty, x \rightarrow 0$ & $\infty$ & $\infty$ & $n-1$ \\
$\mathcal{I}_{\infty}^{3}$ & $(\arctan (n-1), 3 \pi / 2)$ & $y, z \rightarrow-\infty, x \rightarrow 0$ & $\infty$ & $\infty$ & $n-1$ \\
$\mathcal{I}_{\infty}^{4}$ & $(-\arctan (n-1), 3 \pi / 2)$ & $y, z \rightarrow-\infty, x \rightarrow 0$ & $\infty$ & $\infty$ & $n-1$ \\
\hline
\end{tabular}

Table 11. Coordinates, eigenvalues and value of $r^{\prime}$ of the ordinary asymptotic fixed points in non-vacuum $R^{n}$-gravity.

\begin{tabular}{ccccc}
\hline Point & $(\theta, \phi)$ & Eigenvalues $\theta-\phi$ & $r^{\prime}$ & Behavior \\
\hline $\mathcal{A}_{\infty}$ & $(0,0)$ & {$[1,1]$} & 2 & $\left|\mathcal{N}-\mathcal{N}_{\infty}\right|=\left[C_{1} \pm \frac{C_{0}}{2}\left(t-t_{0}\right)\right]^{2}$ \\
$\mathcal{B}_{\infty}$ & $(\pi, \pi)$ & {$[-1,-1]$} & -2 & $\left|\mathcal{N}-\mathcal{N}_{\infty}\right|=\left[C_{1} \pm \frac{C_{0}}{2}\left(t-t_{0}\right)\right]^{2}$ \\
$\mathcal{C}_{\infty}$ & $(\pi / 2,0)$ & {$\left[1, \frac{1}{n-1}\right]$} & -2 & $\left|\mathcal{N}-\mathcal{N}_{\infty}\right|=\left[C_{1} \pm \frac{C_{0}}{2}\left(t-t_{0}\right)\right]^{2}$ \\
$\mathcal{D}_{\infty}$ & $(-\pi / 2, \pi)$ & {$\left[-1, \frac{1}{1-n}\right]$} & 2 & $\left|\mathcal{N}-\mathcal{N}_{\infty}\right|=\left[C_{1} \pm \frac{C_{0}}{2}\left(t-t_{0}\right)\right]^{2}$ \\
$\mathcal{E}_{\infty}$ & $(\pi / 4,0)$ & {$\left[-\frac{\sqrt{2}}{2}, \frac{n \sqrt{2}}{2(n-1)}\right]$} & $-\frac{\sqrt{2}}{2}$ & $a=a_{0} \exp \left[\lambda\left(t-t_{0}\right)\right]$ \\
$\mathcal{F}_{\infty}$ & $(3 \pi / 4, \pi)$ & {$\left[\frac{\sqrt{2}}{2}, \frac{n \sqrt{2}}{2(1-n)}\right]$} & $\frac{\sqrt{2}}{2}$ & $a=a_{0} \exp \left[\lambda\left(t-t_{0}\right)\right]$ \\
$\mathcal{G}_{\infty}$ & $(\pi / 2, \pi / 4)$ & {$\left[\frac{\sqrt{2}}{2(1-n)}, \frac{n \sqrt{2}}{2(n-1)}\right]$} & $\frac{(1-2 n) \sqrt{2}}{2(1-n)}$ & $\left|\mathcal{N}-\mathcal{N}_{\infty}\right|=\left[C_{1} \pm C_{0}\left|\frac{n-1}{2 n-1}\right|\left(t-t_{0}\right)\right]^{\frac{2 n-1}{n-1}}$ \\
$\mathcal{H}_{\infty}$ & $(\pi / 2,5 \pi / 4)$ & {$\left[\frac{\sqrt{2}}{2(n-1)}, \frac{n \sqrt{2}}{2(1-n)}\right]$} & $\frac{(2 n-1) \sqrt{2}}{2(1-n)}$ & $\left|\mathcal{N}-\mathcal{N}_{\infty}\right|=\left[C_{1} \pm C_{0}\left|\frac{n-1}{2 n-1}\right|\left(t-t_{0}\right)\right]^{\frac{2 n-1}{n-1}}$ \\
\hline
\end{tabular}


Table 12. Stability of the ordinary asymptotic fixed points for $R^{n}$-gravity with matter. These results are independent of the value of $w$.

\begin{tabular}{cccc}
\hline Point & $n<0$ & $0<n<1$ & $n>1$ \\
\hline $\mathcal{A}_{\infty}$ & repellor & repellor & repellor \\
$\mathcal{B}_{\infty}$ & attractor & attractor & attractor \\
$\mathcal{C}_{\infty}$ & saddle & saddle & saddle \\
$\mathcal{D}_{\infty}$ & saddle & saddle & saddle \\
$\mathcal{E}_{\infty}$ & saddle & attractor & saddle \\
$\mathcal{F}_{\infty}$ & saddle & repellor & saddle \\
$\mathcal{G}_{\infty}$ & saddle & saddle & saddle \\
$\mathcal{H}_{\infty}$ & saddle & saddle & saddle \\
\hline
\end{tabular}

For the points $\mathcal{E}_{\infty}$ and $\mathcal{F}_{\infty}, y \rightarrow 0$ and $x, z \rightarrow \pm \infty$ at the same rate $(x / z \rightarrow 1)$. For these points equation (54) reduces to $\dot{H}=0$ which corresponds to the de Sitter solution $a=a_{0} \exp \left[\lambda\left(t-t_{0}\right)\right]$ where $\lambda$ is an integration constant.

For the points $\mathcal{G}_{\infty}, \mathcal{H}_{\infty}, z \rightarrow 0$ and $x, y \rightarrow \pm \infty$ at the same rate $(x / y \rightarrow 1)$. In this limit the first and third equation of the system (51) reduce to

$$
x^{\prime} \rightarrow-x^{2},
$$

which admits the solution

$$
x=z \rightarrow \frac{1}{\mathcal{N}-\mathcal{N}_{\infty}} .
$$

Substituting this result in the (54) we obtain

$$
\left|\mathcal{N}-\mathcal{N}_{\infty}\right|=\left[C_{1} \pm C_{0}\left|\frac{n-1}{2 n-1}\right|\left(t-t_{0}\right)\right]^{\frac{2 n-1}{n-1}},
$$

as expected from the fact that for $z=0$ the system (51) reduces to (21).

The parameter $n$ also determines the stability of the ordinary fixed points. As we can see from Table 12, all but the point $\mathcal{B}_{\infty}$ and $\mathcal{E}_{\infty}$ are unstable for every value of $n$. The point $\mathcal{B}_{\infty}$ is always an attractor and the point $\mathcal{E}_{\infty}$ is an attractor only if $0<n<1$.

In addition to the ordinary fixed point, there is a second class of fixed points defined by two "fixed lines" $L 1$ with $\phi=\pi / 2$ and $L 2$ with $\phi=3 \pi / 2$ in the $(\theta, \phi)$ plane. The solution associated with the fixed lines can be obtained with the same method used above. We obtain

$$
\left|\mathcal{N}-\mathcal{N}_{\infty}\right|=\left[C_{1} \pm \frac{C_{0}}{2}\left(t-t_{0}\right)\right]^{2},
$$

For the points on $L 1$ and $L 2$, one of the eigenvalues of the associated linearized matrix is null so that the system is structurally unstable. This means that the linearization is inappropriate and the local phase portrait is determined principally by the nonlinear terms. In the presence of structural instability there is no simple way to devise 
the stability properties of a fixed point and we are forced to study directly small perturbations around the fixed lines. Developing the (66, 67) at first order about the generic point $\left(\pi / 2, \theta_{0}\right)$, we obtain

$$
\begin{aligned}
& \delta \theta^{\prime}=\delta \phi \frac{n \sin ^{2}\left(\theta_{0}\right) \cos \left(\theta_{0}\right)}{n-1}, \\
& \delta \phi^{\prime}=\delta \phi \frac{(n-1) \cos \left(\theta_{0}\right)-\sin \left(\theta_{0}\right)}{1-n} .
\end{aligned}
$$

This system admits the solutions

$$
\begin{aligned}
& \delta \theta= \pm \frac{A_{1} n \sin ^{2}\left(\theta_{0}\right) \cos \left(\theta_{0}\right)}{\sin \left(\theta_{0}\right)+(1-n) \cos \left(\theta_{0}\right)} \exp \left[ \pm \frac{(n-1) \cos \left(\theta_{0}\right)-\sin \left(\theta_{0}\right)}{1-n} \mathcal{N}\right]+B_{1}, \\
& \delta \phi=A_{1} \exp \left[ \pm \frac{(n-1) \cos \left(\theta_{0}\right)-\sin \left(\theta_{0}\right)}{1-n} \mathcal{N}\right],
\end{aligned}
$$

where the " \pm " is connected with the sign of the perturbation $\delta \phi$ and $A_{1}$ and $B_{1}$ are integration constants. Now, if we look at the value of $r^{\prime}$ at the generic point $\left(\pi / 2, \theta_{0}\right)$ we obtain

$$
\left.r^{\prime}\right|_{\phi=\pi / 2}=2 \frac{(n-1) \cos (\theta)-\sin (\theta)}{n-1}
$$

and discover that the stability of the fixed point depends only on the sign of the term $\frac{(n-1) \cos \left(\theta_{0}\right)+\sin \left(\theta_{0}\right)}{n-1}$. If $\mathcal{N}$ increases with time (i.e. the model is expanding) and the perturbation $\delta \phi$ is positive, the points on the fixed line $\phi=\pi / 2$ are saddle nodes for every value of $\theta$ and $n$. Conversely, if the perturbation $\delta \phi$ is negative, the points on the fixed line $\phi=\pi / 2$ are pure attractors if $\arctan (n-1)<\theta<\pi / 2$ for $n>1$ and $-\pi / 2<\theta<\arctan (n-1)$ for $n<1$ and are pure repellors otherwise. This behavior is reversed in case of contraction $(\mathcal{N}$ decreases with $t)$.

Similarly, expanding the equations (66,67) at first order about the generic point $\left(3 \pi / 2, \theta_{0}\right)$ we obtain

$$
\begin{aligned}
\delta \theta^{\prime} & =\delta \phi \frac{n \sin ^{2}\left(\theta_{0}\right) \cos \left(\theta_{0}\right)}{1-n} \\
\delta \phi^{\prime} & =\delta \phi \frac{\sin \left(\theta_{0}\right)+(n-1) \cos \left(\theta_{0}\right)}{1-n} .
\end{aligned}
$$

This system admits the solutions

$$
\begin{aligned}
& \delta \theta= \pm \frac{A_{2} n \sin ^{2}\left(\theta_{0}\right) \cos \left(\theta_{0}\right)}{\sin \left(\theta_{0}\right)+(n-1) \cos \left(\theta_{0}\right)} \exp \left[ \pm \frac{\sin \left(\theta_{0}\right)+(n-1) \cos \left(\theta_{0}\right)}{1-n} \mathcal{N}\right]+B_{2}, \\
& \delta \phi=A_{2} \exp \left[ \pm \frac{\sin \left(\theta_{0}\right)+(n-1) \cos \left(\theta_{0}\right)}{1-n} \mathcal{N}\right],
\end{aligned}
$$

where the " \pm " is connected with the sign of the perturbation $\delta \phi$ and $A_{2}$ and $B_{2}$ are integration constants. Again, if we look at the value of $r^{\prime}$ at the generic point $\left(\pi / 2, \theta_{0}\right)$

$$
\left.r^{\prime}\right|_{\phi=3 \pi / 2}=2 \frac{(n-1) \cos (\theta)+\sin (\theta)}{n-1},
$$

we discover that the stability of the fixed point depends only on the sign of the term $\frac{(n-1) \cos \left(\theta_{0}\right)+\sin \left(\theta_{0}\right)}{n-1}$. If $\mathcal{N}$ increases with time (i.e. the model is expanding) the 
perturbation $\delta \phi$ is positive, the points of the fixed line $\phi=3 \pi / 2$ are saddle nodes for every value of $\theta$ and $n$. Conversely, if the perturbation $\delta \phi$ is negative, the points of the fixed line $\phi=3 \pi / 2$ are pure attractors if $-\pi / 2<\theta<\arctan (1-n)$ for $n>1$ and $\arctan (1-n)<\theta<\pi / 2$ for $n<1$ and are pure repellors otherwise. This behavior is reversed in case of contraction.

Finally, there are four special points given by $( \pm \arctan (n-1), \pi / 2+k \pi)$ with $k=0,1$. These points belong to the lines $L 1$ and $L 2$ and are associated with the solutions (70). The difference is that, in calculating their stability we find that both the eigenvalues are null. This situation is more complex than the previous one, and the stability analysis requires us to perturb the system at the second order. Unfortunately, the solution of this system turns out to be achievable only numerically and hence makes it impossible to give a general stability analysis. For this reason we will limit ourselves to giving only plots of the solutions for the point $(\pi / 2, \arctan (n-1))$ and for a set of specific values of $n$ (see figures 9-14). It is clear that for all the values of $n$ the perturbation on $\theta$ approaches a constant. Such a behavior is due to the fact that the fixed lines are themselves invariant submanifolds and therefore small perturbations on $\theta$ induce the state to evolve on the line towards the nearest attractor.

It is clear that the global behavior of the non-vacuum $R^{n}$-gravity cosmological models is not trivial. We have three different types of transient states which represent, Milne and "Friedman-like" solutions. As in the vacuum case, for values of $n$ far from one the power law solution $\mathcal{C}$ is an attractor. When $n$ approaches unity, other attractors i.e. $\mathcal{D}$ come into play and the stability of $\mathcal{C}$ changes dramatically. Asymptotically two different sets of attractors are present. The first one is made up of ordinary points i.e. $\mathcal{B}_{\infty}$ and, for $0<n<1, \mathcal{E}_{\infty}$; the second one by the $\operatorname{arcs}(\phi=\pi / 2,0<\theta<\arctan (n-1))$ and $(\phi=3 \pi / 2, \arctan (1-n)<\theta<0)$ for $n>1$ and $\arctan (n-1)<\theta<0$ and $0<\theta<\arctan (1-n)$ for $n<1$.

The most interesting scenario is an orbit that approaches first the $\mathcal{G}$ point and then the $\mathcal{C}$ solution. Clearly, the values of $n$ should ensure $\mathcal{G}$ is physical and not too different from the standard Friedmann solution in order be consistent with observational constraints. At the same time, $n$ has to be chosen to ensure that $\mathcal{C}$ is associated with an accelerating solution in order to get a late acceleration phase. Moreover, since the planes $y=0$ and $z=0$ are invariant submanifolds and cannot be crossed by any orbit, we have to make sure that the $y$ coordinate of the points $\mathcal{C}$ and $\mathcal{G}$ have the same sign. Using the Tables given above, we can select the value of $n$ for which this is realized:

$$
\begin{array}{ll}
w=0,1 / 3 \quad\left\{\begin{array}{cll}
n \lesssim 0.37 & \text { if } & n \in \mathcal{N}_{\text {even }} \\
1.37 \lesssim n \lesssim 2 & \text { if } & n \in \mathcal{N}_{\text {odd }}
\end{array}\right. \\
w=1 & \left\{\begin{array}{ccc}
n \lesssim 0.37 & \text { if } & n \in \mathcal{N}_{\text {even }} \\
1.5 \lesssim n \lesssim 2 & \text { if } & n \in \mathcal{N}_{\text {odd }}
\end{array}\right.
\end{array}
$$




\section{Discussion and Conclusions}

In this paper, we have given a detailed analysis of the behavior of $R^{n}$-gravity cosmological models using the dynamical system approach. Defining an effective fluid representing the higher order corrections to standard GR, it is possible to define a system of first order differential equations which are equivalent to the cosmological equations. In this way, using standard dynamical systems theory, we have obtained a qualitative description of the global and asymptotic behavior of these cosmologies. This allows to achieve a comprehensive (but qualitative) description of the global features of such models.

Our investigation began with an analysis of the the pure gravity case in which the phase space is two dimensional. We found five fixed points, two of them (one of which is double) with coordinates independent of the parameter $n$ and two whose position on the plane changes with $n$. These fixed points correspond to the four exact solutions listed in Table 1. One of them, namely the solution associated with the point $\mathcal{C}$, is particularly interesting because it can give rise to accelerated expansion. This solution has already been found by solving the field equations directly [10] and was compared with observational data. Our analysis shows that, for the values of $n$ compatible with observations, the solution $\mathcal{C}$ is an attractor.

However, the structure of the phase space shows that once we have set the initial condition, the sign of the Ricci scalar is preserved. In other words there is no way in which these models can evolve from a state $R>0$ to a state $R<0$ or vice versa. Such a transition would be impossible in GR because $R$ is proportional to the energy density and a change in its sign would imply a violation of the energy conditions. In $R^{n}$ gravity, and in a general $f(R)$ gravity, this is not necessarily true because of the more complicated relation between $R$ and $\rho$. However, the main consequence of such a feature is that, in the vacuum phase space, no global attractor can exist and, in particular, $\mathcal{C}$ is an attractor only for specific initial conditions.

The introduction of the matter term in the field equations induces strong constraints on the parameters. The value of $n$ has to be either a relative or an odd denominator rational number to ensure self-consistency of the theory. This was expected: not all forms of non-minimal coupling are necessarily compatible with standard matter. Other constraints come from the fact that the matter energy density is a function of $n$ and is not positive definite in general.

The analysis of the dynamical system reveals that four of the seven fixed points found in this case are generalizations of the fixed point of the vacuum case and present the same stability properties. This implies that, for example the constraints on $n$ found in [16] hold also in this case. The last three fixed points are just transients that, providing $w$ lies in the Zel'dovic interval, are physical only for specific values of $n$. The fixed point $\mathcal{G}$ is particularly interesting and may be interpreted as corresponding to an "almost-Friedmann" transient phase in the evolution of the cosmological model.

We found values of $n$ for which the universe undergoes a transient almost- 
Friedmann behavior, finally evolving into an accelerating phase. The existence of such a transient would not only explain the recently observed non constancy of the deceleration parameter [31], but would also allow, in principle, a period of structure formation. Such a feature is relevant because it is not easy to explain how large scale structure is formed in accelerating universes.

In the asymptotic regime $R^{n}$-gravity possesses number of fixed points which is the same or even bigger than the number of finite ones. In the vacuum case, we found that these fixed points represent essentially an exponential evolution. In two cases the character of the solution (expanding or contracting) depends on the value of the parameter $n$. For $n$ in the interval suggested by the observations we have two attractors for $n<1$ and only one for $n>1$.

In the matter case, the asymptotic structure is very rich. We have two different sets of critical points. One of them is made by single points and one is made by two continuous subsets of the phase space (the "fixed lines"). For the set of points $\left(\mathcal{A}_{\infty}\right.$ $\left.\mathcal{G}_{\infty}\right)$ the asymptotic behaviors are of the same type of the vacuum case apart a pure de Sitter evolution when $x$ and $z$ diverge at the same rate $\left(\mathcal{E}_{\infty}-\mathcal{F}_{\infty}\right)$. For the fixed lines the behavior is of the same type of $\left(\mathcal{A}_{\infty}-\mathcal{D}_{\infty}\right)$.

The results presented in this paper have two key features. Firstly, the application of the dynamical system approach to higher order gravity cosmology seems to be a powerful tool, revealing global features of these models. Secondly, the results seem to indicate that the idea of curvature quintessence i.e. to model the accelerated expansion phenomenon as a high order gravitational effect, not only seems viable, but is also in good agreement with our present understanding of the history of the universe.

\section{References}

[1] Kerner R 1982 Gen. Relativ. Grav. 14453 ; Duruisseau J P, Kerner R 1986 Class. Quantum Grav. 3817

[2] Teyssandier P 1989 Class. Quantum Grav. 6219

[3] Magnano G, Ferraris M and Francaviglia M 1987 Gen. Relativ. Grav. 19465.

[4] Birrell N, Davies P 1982 Quantum Fields in Curved Space (Cambridge: Cambridge Univ. Press)

[5] Green M, Schwarz J and Witten E 1987Superstring theory Vol. 1 \& 2(Cambridge Univ. Press); Polchinski J 1998 String Theory Vol. I \& II (Cambridge Univ. Press)

[6] Maeda K and Ohta N 2004 Phys. Lett. B 597400 Preprint hep-th/0405205 Ohta N 2003 Phys. Rev. Lett. 91061303 Preprint hep-th/0303238 N. Ohta Prog. Theor. Phys. 110 (2003) 269, hep-th/0304172 Ohta N, 2003 Prog. Theor. Phys. 110269 Preprint hep-th/0304172

[7] Buchbinder I L et al. Buchbinder I L 1989 Fortschr. Phys. 37 207; Buchbinder I L 1989 Phys. Lett. B 216 127; Buchbinder I L 1989 Yad. Fiz. (J. NUcl. Phys.) 49 876; Sahpiro I L 1989 Class. Quantum Grav. 61197

[8] Salam A, Strathdee J 1978 Phys. Rev. D 18 4480; Tomboulis E T Phys. Lett. B 70 361; Antoniadis I, Tomboulis E T 1986 Phys. Rev. D33 2756

[9] Maartens R 2004 Liv. Rev. Rel.

[10] Capozziello S 2002 Int. Journ. Mod. Phys. D 11 483; Capozziello S, Carloni S and Troisi A 2003 Recent Res. Devel.Astronomy \& Astrophysics 1 (Capozziello S, Carloni S and Troisi A Preprint 2003 astro-ph/0303041)

[11] Allemandi G, Borowiec A and Francaviglia M 2004 Phys. Rev. D 70043524 
[12] Carrol S M et al. Preprint astro-ph/0306438; Carrol S M et al. Preprint astro-ph/0410031; Vollick D N 2003 Phys. Rev. D 68063510

[13] Flanagan E E 2004 Phys. Rev. Lett. 92 071101; Flanagan E E 2003 Class. Quantum Grav. 21 417 ;

[14] Nojiri S, Odintsov SD 2003Phys. Lett. B 576 5-11; Nojiri S, Odintsov SD 2004 Mod. Phys. Lett. A 19(8) 627; Nojiri S, Odintsov SD 2003 Phys. Rev. D 68 123512; Abdalla M, Nojiri S and Odintsov S D Preprint hep-th/0409177

[15] de Bernardis P et al.2000 Nature 404 955; Stompor R et al.2001 Ap. J. 561 L7; Spergel DN et al.2003 Astrophys. J. Suppl. 148175.

[16] Capozziello S 2003 et al.Int. Journ. Mod. Phys. D 121969

[17] Dynamical System in Cosmology edited by Wainwright J and Ellis G F R (Cambridge: Cambridge Univ. Press 1997) and references therein

[18] Wands D and Holden D J 1998 Class. Quantum Grav. 15 3271; Copeland E J, Liddle A R and Wands D 1998 Phys. Rev. D 574686

[19] Coley A A Gen. Relativ. Grav. 1999311295

[20] E. Gunzig et al. 2000 Class. Quant. Grav. 171783

[21] Landau L D, Lifshitz E M 1951 The Classical Theory of Fields (Oxford: Pergamon Press 1951)

[22] Capozziello S, de Ritis R and Marino A A 1998 Gen. Relativ. Grav. 301247

[23] De Witt B Dynamical Theory of Groups and Fields (New York: Gordon and Breach 1965).

[24] Eddington A S The mathematical theory of relativity (Cambridge: Cambridge Univ. Press 1952)

[25] Lovelock D, Rund H Tensors Differential Forms and Variational Principles ( New York: Dover 1989).

[26] Maarteens R, Taylor D R 1994 Gen. Relativ. Grav. 26599 (1994);

[27] Schmidt H J 1990 Class. Quantum Grav. 7 1023; Schmidt H J 1996 Phys. Rev. D 547906 ; Capozziello S, de Ritis R, Rubano C and Scudellaro P 1996Il Nuovo Cim. 4; Vilenkin A 1985 Phys. Rev. D 322511

[28] Starobinsky A A 1980 Phys. Lett. B 91 99; Müller V, Schmidt H J 1991 Fortschr. Phys. 39 319; S. Capozziello S, Occhionero F and Amendola L 1993 Int. Journ. Mod. Phys. D 1 615; Mijic M B, Morris M S and Suen W M 1986 Phys. Rev. D 342934

[29] reference [17; Goliath M and Ellis G F R 1999 Phys. Rev. D 60023502

[30] Hartman P Ordinary differential equations (New York: Wiley 1964)

[31] Riess A G et al.Ap. J. 607665 (2004) 